\title{
CHIMIE AU LYCÉE ET ENEM: UNE COMPARAISON DES PROGRAMMES
}

\section{ARTICLE ORIGINAL}

GORTZ, Julia Santana ${ }^{1}$, TATY, Salvador Rodrigues², FECURY, Amanda Alves ${ }^{3}$, DENDASCK, Carla Viana ${ }^{4}$, OLIVEIRA, Euzébio de ${ }^{5}$, DIAS, Claudio Alberto Gellis de Mattos $^{6}$

GORTZ, Julia Santana. Et al. Chimie au lycée et Enem: une comparaison des programmes. Revista Científica Multidisciplinar Núcleo do Conhecimento. Année 06, éd.03, vol. 03, pp. 89-99. Mars 2021. ISSN: 2448-0959, Lien d'accès: https://www.nucleodoconhecimento.com.br/education-fr/comparaison-desprogrammes, DOI: 10.32749/nucleodoconhecimento.com.br/educationfr/comparaison-des-programmes

\section{ABSTRAIT}

Le examen national du lycée (ENEM) est une évaluation composée d'une dissertation et de questions à choix multiples. Cette évaluation vise à mesurer les connaissances acquises au lycée. Le but de cet article est de comparer le contenu des questions de la composante Chimie de l'Examen National du Lycée (ENEM) entre les années 2014 à 2018 avec le contenu du programme du cours de chimie

\footnotetext{
${ }^{1}$ Etudiant du Cours Technique de Chimie (lycée) à l'Institut d'Enseignement de Base, Technique et Technologique d'Amapá (IFAP).

${ }^{2}$ Chimiste, Master en Chimie (UFMA), Professeur et Chercheur dans le Cours de Chimie à I'Institut de Base, Technique et Technologique d'Amapá (IFAP), Coordinateur du Cours Technique de Chimie (IFAP).

${ }^{3}$ Biomédical, doctorat en maladies tropicales (UFPA), professeur et chercheur du cours de médecine au Campus Macapá, Université fédérale d'Amapá (UNIFAP).

${ }^{4}$ Théologien, docteur en psychanalyse, chercheur au Centre de Recherche et d'Etudes Avancées - CEPA.

${ }^{5}$ Biologiste, docteur en maladies tropicales (UFPA), professeur et chercheur au cours d'éducation physique à I'Université fédérale du Pará (UFPA).

${ }^{6}$ Biologiste, docteur en théorie et recherche du comportement (UFPA), professeur et chercheur du cours de chimie à l'Institut fondamental, technique et technologique d'Amapá (IFAP) et du programme d'études supérieures en enseignement professionnel et technologique (PROFEPT IFAP).
}

RC: 78616

Disponível em: https://www.nucleodoconhecimento.com.br/education- 
technique à I'Institut Fédéral d'Amapá (IFAP) . La recherche a été menée à l'aide de questions de chimie de l'examen national du lycée (ENEM) tirées du programme Super Professor (logiciel). Le contenu enseigné au cours des trois années du cours technique de chimie à I'Institut fédéral d'Amapá (IFAP) répond aux exigences de l'examen national du lycée (ENEM). La charge de travail est également suffisante pour le développement des disciplines de base et techniques. L'analyse du contenu démontre que, comme il s'agit d'un cours technique, il fournit des connaissances approfondies, ce qui augmente la subvention pour la réalisation de l'ENEM. Ce contenu est formé par la théorie et aussi par une grande expérience pratique (laboratoire). Les connaissances pratiques aident énormément à la fixation de l'apprentissage et fournissent des connaissances pour discuter du contenu.

Mots clés: Enseignement, Chimie, ENEM, EPT, Curriculum.

\section{INTRODUCTION}

Le examen national du lycée (ENEM) est une évaluation composée d'une dissertation et de questions à choix multiples. Les questions correspondent aux domaines des Sciences Humaines et de ses Technologies (Histoire, Géographie, Philosophie et Sociologie |); Sciences naturelles et leurs technologies (physique, chimie et biologie); Langues, codes et leurs technologies (portugais, langue étrangère, arts et éducation physique); et les mathématiques et ses technologies (mathématiques). Cette évaluation vise à mesurer les connaissances acquises au lycée (Moretto et Wittke, 2018).

Un institut fédéral est une institution dont l'objectif est de répondre au besoin de main-d'œuvre technique qualifiée, afin que les étudiants soient mieux préparés à acquérir à la fois des connaissances de base et des connaissances techniques (Alves et al., 2013; Penha et al., 2020). II existe actuellement 38 instituts fédéraux présents dans tous les États (Brésil, 2018).

RC: 78616

Disponível em: https://www.nucleodoconhecimento.com.br/education- 
En 2007, le 25 octobre, l'École technique fédérale d'Amapá (ETFAP) a été créée. Le 29 décembre 2008, en raison de la loi 11892, I'ETFAP est transformé en Institut fédéral d'éducation, de science et de technologie d'Amapá (IFAP) (Brésil, 2019; Marques et al., 2020).

Son public cible est $50 \%$ du niveau lycée, cours technique lié au lycée (intégré, subséquent et concomitant); $30 \%$ des diplômes d'enseignement supérieur, de licence et de technologie et $20 \%$ pour les diplômes de premier cycle. Ayant également des études de troisième cycle: Lato Sensu et Stricto Sensu (Brésil, 2019; Castro et al., 2020).

Le cours de chimie a été ouvert en 2017, avec 3 ans à temps plein. Sa fonction est de former des techniciens capables d'opérer, de contrôler et de surveiller les processus industriels et de laboratoire. Maintenir le contrôle de la qualité des matières premières, des intrants et des produits. Effectuer des échantillons, des analyses chimiques, physico-chimiques et microbiologiques. Développer des processus et des produits. Les professionnels formés dans ce cours ont les domaines d'activité suivants: industries chimiques; Laboratoires de contrôle de la qualité, de certification des produits chimiques, alimentaires et connexes (Brésil, 2019a).

Au lycée technique de chimie proposé par l'Institut fédéral d'Amapá, le menu prévoit pour la première année: chimie générale (chimie dans notre vie quotidienne, évolution des modèles atomiques et des liaisons chimiques, fonctions inorganiques; problèmes environnementaux et réactions chimiques) et matériaux techniques (chimie expérimentale, chimie inorganique et chimie organique). Dans la deuxième année, il est vu: chimie générale (solutions, réactions exothermiques et endothermiques, vitesse, facteurs, équilibre et déplacement et électrochimie) et des questions techniques (physico-chimique, chimie analytique, analyse instrumentale et corrosion). Enfin en troisième année: chimie générale (chimie organique, hydrocarbures, fonctions oxygénées, azotées et mixtes et propriétés organiques) et

RC: 78616

Disponível em: https://www.nucleodoconhecimento.com.br/education- 
matériaux techniques (pétrole et polymères, procédés chimiques industriels et technologie des biocarburants) (Brésil, 2016).

Le contenu des questions de chimie d'Enem est divisé en 10 unités principales, qui sont: les transformations chimiques (où sont inclus les sujets du numéro atomique, du nombre de masse, des isotopes, de la masse atomique, du tableau périodique et des réactions chimiques); Représentation des transformations chimiques (sujet qui comprend l'équation d'équations chimiques et les calculs stœchiométriques); Matériaux, leurs propriétés et utilisations (qui comprend les propriétés des matériaux, les états physiques des matériaux, les changements d'état, les mélanges et les forces intermoléculaires); Eau (qui comprend les solutions, les acides, les bases, les sels et les oxydes, la nomenclature et les indicateurs); Transformations chimiques et énergie (où des contenus tels que l'enthalpie, les équations thermochimiques, la loi de Hess, la cellule et l'électrolyse sont insérés); Dynamique des transformations chimiques (qui comprend la vitesse de réaction et l'énergie d'activation); Transformation chimique et équilibre (qui comprend les sujets de la constante d'équilibre, de l'équilibre acido-basique et $\mathrm{du} \mathrm{pH}$, de la solubilité du sel et de l'hydrolyse); Composés de carbone (comprend les fonctions organiques, les hydrocarbures et les polymères); Relations de la chimie avec les technologies, la société et l'environnement (y compris le sujet de la pollution); Les énergies chimiques dans la vie de tous les jours (inclut les sujets du pétrole, du gaz naturel et du charbon) (Brasil, 2015).

\section{BUTS}

Comparez le contenu des questions de la composante de chimie de l'Examen national du secondaire (ENEM) entre les années 2014 et 2018 avec le contenu du programme du cours de chimie technique à l'Institut fédéral d'Amapá (IFAP).

RC: 78616

Disponível em: https://www.nucleodoconhecimento.com.br/education- 


\section{MÉTHODOLOGIE}

La recherche a été réalisée à l'aide de questions de chimie de l'examen national du lycée (ENEM) tirées du programme Super Professor (logiciel), acquis auprès d'Interbits Informática (https://www.sprweb.com.br/mod_superpro/index.php) . La discipline chimique et les années 2014 à 2018 ont été sélectionnées dans le programme. Les questions ont été classées selon le programme. Par la suite, une comparaison a été faite entre ceux-ci et le programme de la composante chimique du cours technique de l'Institut fédéral d'Amapá (IFAP). La recherche bibliographique a été menée sur des articles scientifiques, sur des ordinateurs de l'Institut de l'Institut fédéral de l'éducation, des sciences et de la technologie d'Amapá, Campus Macapá, situé à Rodovia BR 210 KM 3, s / n - Bairro Brasil Novo. CEP: 68.909-398, Macapá, Amapá, Brésil. Les données ont été compilées dans l'application Excel, qui fait partie de la suite Office de Microsoft Corporation.

\section{RÉSULTATS}

Le tableau 1 montre le contenu des questions de chimie ENEM entre 2014 et 2018, par quantité et pourcentage de questions. Ces questions correspondent à $78,82 \%$ du total des questions de la période. Les questions sur les enzymes, les glucides, les indicateurs, les changements d'état, les symboles, la pollution et les propriétés physico-chimiques n'apparaissent pas dans la période $(0,00 \%)$. Les questions sur la structure de l'atome, la cinétique chimique, la densité, les propriétés colligatives, les solutions, la classification périodique, l'équation et l'équilibre de la réaction, les fonctions inorganiques, les pratiques de laboratoire, la substance et le mélange, les chaînes carbonées, l'isomérie plate, l'huile et les polymères apparaissent à 1,18\%. Les questions sur les concentrations de solution, l'équilibre chimique ou ionique, I'hydrolyse du sel, l'oxydoréduction et les fonctions sont de 2,35\%. Les questions sur les liaisons chimiques et la géométrie moléculaire, la radioactivité et l'isomérie spatiale correspondent à $3,53 \%$. Le calcul stoechiométrique et la séparation des

RC: 78616

Disponível em: https://www.nucleodoconhecimento.com.br/education- 
mélanges correspondent à $4,71 \%$ des questions. Les questions sur la thermochimie et les réactions organiques sont de $7,06 \%$. Les forces intermoléculaires et électrochimiques apparaissent à $8,24 \%$.

RC: 78616

Disponível em: https://www.nucleodoconhecimento.com.br/educationfr/comparaison-des-programmes 
Tableau 1 Classification du contenu des questions de chimie ENEM entre 2014 et 2018, par quantité et pourcentage de questions.

\begin{tabular}{|c|c|c|}
\hline \multicolumn{3}{|c|}{ Classificaçăo do autor após analise de conteúdo } \\
\hline Conteúdo de Química & Quantidade & Porcentagem \\
\hline Enzimas & 0 & $0.00 \%$ \\
\hline Hidratos de Carbono & 0 & $0.00 \%$ \\
\hline Indicadores & 0 & $0.00 \%$ \\
\hline Mudanças de Esta do & 0 & $0.00 \%$ \\
\hline Símbolos & 0 & $0.00 \%$ \\
\hline Poluição & 0 & $0.00 \%$ \\
\hline Proprieda des Físico-Químicas & 0 & $0.00 \%$ \\
\hline Es trutura do atomo & 1 & $1.18 \%$ \\
\hline Cinética Química & 1 & $1.18 \%$ \\
\hline Densidade & 1 & $1.18 \%$ \\
\hline Proprieda des Coligativas & 1 & $1.18 \%$ \\
\hline Soluções & 1 & $1.18 \%$ \\
\hline Class ificação Periódica & 1 & $1.18 \%$ \\
\hline Equacionamento e Balanceamento de Reações & 1 & $1.18 \%$ \\
\hline Funções Inorgânicas & 1 & $1.18 \%$ \\
\hline Práticas de Laboratório & 1 & $1.18 \%$ \\
\hline Substância e Mistura & 1 & $1.18 \%$ \\
\hline Cadeias Carbônicas & 1 & $1.18 \%$ \\
\hline Is omeria Plana & 1 & $1.18 \%$ \\
\hline Petróleo & 1 & $1.18 \%$ \\
\hline Polímeros & 1 & $1.18 \%$ \\
\hline Concentrações das Soluçôes & 2 & $2.35 \%$ \\
\hline Equilíbrio Químico ou lônico & 2 & $2.35 \%$ \\
\hline Hidrólise de Sa is & 2 & $2.35 \%$ \\
\hline Óxido-redução & 2 & $2.35 \%$ \\
\hline Funções & 2 & $2.35 \%$ \\
\hline Ligações Químicas e Geometria Molecular & 3 & $3.53 \%$ \\
\hline Radioatividade & 3 & $3.53 \%$ \\
\hline Is omeria Espacial & 3 & $3.53 \%$ \\
\hline Cálculo Estequiométrico & 4 & $4.71 \%$ \\
\hline Separação de Misturas & 4 & $4.71 \%$ \\
\hline Termoquímica & 6 & $7.06 \%$ \\
\hline Reações Orgânicas & 6 & $7.06 \%$ \\
\hline Forças intermoleculares & 7 & $8.24 \%$ \\
\hline Eletroquímica & 7 & $8.24 \%$ \\
\hline
\end{tabular}

RC: 78616

Disponível em: https://www.nucleodoconhecimento.com.br/education- 
Le tableau 2 montre le chevauchement du contenu des questions de chimie ENEM entre 2014 et 2018, par quantité et pourcentage de questions. Les questions qui se chevauchent représentent $21,18 \%$ du total de l'examen sur la période. Les questions Forces intermoléculaires + Propriétés physico-chimiques, Forces intermoléculaires + Propriétés colligatives, Forces intermoléculaires + Séparation des mélanges, Liaisons chimiques et géométrie moléculaire + classification périodique, Hydrolyse des sels + Équation et bilan des réactions + Fonctions inorganiques, Balance chimique ou ionique + Polymères, Glucides + Oxydo-réduction, Hydrolyse des sels + Indicateurs, Symboles + Substance et mélange, Équation et bilan des réactions + Pollution et Pollution + Les chaînes carbonées apparaissent à 1,18\%. Les problèmes Enzymes + Réactions organiques, Concentrations de solutions + Solutions représentent $2,35 \%$.

Tableau 2 Classification du contenu de chevauchement des questions de chimie ENEM entre 2014 et 2018, par quantité et pourcentage de questions. Les questions qui se chevauchent représentent $21,18 \%$

\begin{tabular}{l|c|c|}
\hline \multicolumn{2}{c}{ Classificação do autor após analise de conteúdo sobre posto } \\
\hline \multicolumn{1}{c|}{ Conteúdo de Química } & Quantidade & Porcentagem \\
\hline Forças intermoleculares + Propriedades Fisico-Quimicas & 1 & $1.18 \%$ \\
\hline Forças intermoleculares + propriedades coligativas & 1 & $1.18 \%$ \\
\hline Forças Intermoleculares + Separação de Misturas & 1 & $1.18 \%$ \\
\hline Forças Intermolecula res + Mudanças de Estado & 1 & $1.18 \%$ \\
\hline Ligações Químicas e Geometria Molecular + Classificação Periódica & 1 & $1.18 \%$ \\
\hline $\begin{array}{l}\text { Hidrólise de Sais + Equacionamento e Balanceamento de Reações + } \\
\text { Funções Inorgânicas }\end{array}$ & 1 & $1.18 \%$ \\
\hline Equilíbrio Químico ou lônico + Polímeros & 1 & $1.18 \%$ \\
\hline Hidratos de Carbono + Óxido-redução & 1 & $1.18 \%$ \\
\hline Hidrólise de Sais + Indicadores & 1 & $1.18 \%$ \\
\hline Simbolos + Substância e Mistura & 1 & $1.18 \%$ \\
\hline Equacionamento e Balanceamento de Reações + Poluição & 1 & $1.18 \%$ \\
\hline Poluição + Cadeias Carbônicas & 1 & $1.18 \%$ \\
\hline Enzimas + Reações Orgânicas & 2 & $2.35 \%$ \\
\hline Concentrações das Soluções + Soluções & 2 & $2.35 \%$ \\
\hline Cadeias Carbônicas + Reações Orgânicas & 2 & $2.35 \%$ \\
\hline
\end{tabular}

RC: 78616

Disponível em: https://www.nucleodoconhecimento.com.br/education- 
Les matières du menu de chimie du cours technique de chimie de la FIPA, par année et par unité (se référant à chaque période de deux mois), sont présentées dans le tableau 3. Dans le cours de chimie, les matières sont réparties entre le lycée normal et les matières techniques. sujets spécifiques au cours. Dans les disciplines du programme standard, la composante utilisée au cours des trois années est la chimie générale. Sa charge de travail totale est de 240 heures, 80 heures par an. La première année, votre première unité est donnée en 15 heures, la deuxième unité en 20 heures, la troisième en 25 heures et la quatrième en 20 heures. La deuxième année, le premier contenu est donné en 15 heures, le deuxième contenu en 15 heures, le troisième en 25 heures et le quatrième en 25 heures. La troisième année, la première unité a une charge de travail de 15 heures, la deuxième unité a une charge de travail de 20 heures, la troisième de 30 heures et la quatrième de 15 heures.

Les autres matières (curriculaires techniques) mentionnées dans le tableau 3 sont des matières techniques spécifiques au cours, elles sont semestrielles et ont une charge de travail totale de 80 heures par semestre chacune. Au cours de la première année, les sujets sont cités: chimie expérimentale, qui a une charge de travail de 10 heures, 20 heures, 25 heures et 25 heures pour les unités un, deux, trois et quatre respectivement; chimie inorganique, avec une charge de travail de 15 heures pour la première unité, 15 heures pour la deuxième unité, 25 heures pour la troisième unité et 25 heures pour la quatrième unité; chimie organique, dont la première unité est donnée en 25 heures, la deuxième unité en 15 heures, la troisième en 20 heures et la quatrième en 20 heures. En deuxième année, les matières techniques sont les suivantes: Physico-chimie, avec sa première unité donnée en 25 heures, sa deuxième unité en 20 heures, la troisième en 20 heures et la quatrième en 15 heures; chimie analytique, avec 18 heures pour sa première unité, 20 heures pour la deuxième unité, 23 heures pour la troisième unité et 19 heures pour la quatrième unité; l'analyse instrumentale, avec ses quatre unités, est donnée en respectivement 10 heures, 20 heures, 25 heures et 25 heures; corrosion, où votre première unité est

$\mathrm{RC}: 78616$

Disponível em: https://www.nucleodoconhecimento.com.br/education- 
donnée en 10 heures, votre deuxième unité est donnée en 25 heures, votre troisième unité est donnée en 20 heures et votre quatrième en 25 heures. Au cours de la troisième année, les disciplines spécifiques mentionnées dans le tableau sont: I'huile et les polymères, leurs unités étant respectivement données en 18 heures, 22 heures, 22 heures et 18 heures; les procédés chimiques industriels, avec sa première unité donnée en 25 heures, la deuxième unité en 20 heures, la troisième unité en 20 heures et la quatrième en 15 heures; la technologie des biocarburants, avec une charge de travail de 25 heures pour la première unité, 25 heures pour la deuxième unité, 10 heures pour la troisième et 20 heures pour la quatrième.

RC: 78616

Disponível em: https://www.nucleodoconhecimento.com.br/education- 
Tableau 3. Sujets du menu chimie du cours technique de chimie à la FIPA, par année et par unité.

DISGPLINA CURRICULAR PADRĀO

\begin{tabular}{|c|c|c|c|c|c|c|}
\hline \multirow[b]{2}{*}{ Unidade } & \multicolumn{6}{|c|}{ Ano } \\
\hline & $1^{\circ} \mathrm{ANO}$ & $\begin{array}{c}n^{\circ} \text { de } \\
\text { horas aula }\end{array}$ & $2^{\circ} \mathrm{ANO}$ & de horas at & $3^{\circ} \mathrm{ANO}$ & $\begin{array}{c}\mathbf{n}^{\circ} \text { de } \\
\text { horas aula }\end{array}$ \\
\hline I & $\begin{array}{c}\text { A Quimica emnosso } \\
\text { cotidiano }\end{array}$ & 15 horas & Solbções & 15 horas & Quimica Orgânica & 15 horas \\
\hline II & $\begin{array}{l}\text { A evohyção dos } \\
\text { modelos atôricos e } \\
\text { ligações Químicas. }\end{array}$ & 20 horas & $\begin{array}{c}\text { Reações exotémicas } \\
\text { e endotérmicas }\end{array}$ & 15 horas & Hidrocarb onetos & 20 horas \\
\hline III & $\begin{array}{c}\text { Funções Inorgânicas } \\
\text { e problemas } \\
\text { anbientais }\end{array}$ & 25 horas & $\begin{array}{c}\text { Velocidiade, fatores, } \\
\text { equilibrio e } \\
\text { deslocamento }\end{array}$ & 25 horas & $\begin{array}{l}\text { Funções Oxigenadas, } \\
\text { nitrogenadas e mis tas }\end{array}$ & 30 horas \\
\hline IV & Reações Quimicas & 20 horas & Eletro-Quimica & 25 horas & $\begin{array}{c}\text { Propriedades } \\
\text { Orgânicas }\end{array}$ & 15 horas \\
\hline & & 80 horas & & 80 horas & & 80 horas \\
\hline
\end{tabular}

DISCIPLINA CURRICULAR TÉCNICA - 19 ANO

\begin{tabular}{|c|c|c|c|c|c|c|}
\hline \multirow[b]{2}{*}{ Unidade } & \multicolumn{6}{|c|}{ Ano } \\
\hline & $\begin{array}{c}\text { Quimica } \\
\text { Experimental }\end{array}$ & $\begin{array}{c}\mathbf{n}^{\circ} \text { de } \\
\text { horas aula }\end{array}$ & $\begin{array}{l}\text { Quimica } \\
\text { Inorgânica }\end{array}$ & $\begin{array}{c}\mathbf{n}^{\circ} \text { de } \\
\text { horas aula }\end{array}$ & $\begin{array}{l}\text { Quimica } \\
\text { Orgânica }\end{array}$ & $\begin{array}{c}\mathbf{n}^{\circ} \text { de } \\
\text { horas aula }\end{array}$ \\
\hline I & $\begin{array}{c}\text { Introdução a os } \\
\text { trabalhos em } \\
\text { laboratório. }\end{array}$ & 10 horas & $\begin{array}{c}\text { Quimica dos não } \\
\text { metais }\end{array}$ & 15 horas & $\begin{array}{c}\text { Introdução a } \\
\text { mecanismo de reação }\end{array}$ & 25 horas \\
\hline II & $\begin{array}{c}\text { Obtenção e us o de } \\
\text { calor }\end{array}$ & 20 horas & Quimica dos metais & 15 horas & Ácidos e Bases & 15 horas \\
\hline III & $\begin{array}{c}\text { Process os de } \\
\text { separação de mis turas }\end{array}$ & 25 horas & $\begin{array}{l}\text { Compostos de } \\
\text { coordenação }\end{array}$ & 25 horas & $\begin{array}{c}\text { Reações de adição- } \\
\text { eliminação } \\
\text { nucleoflica em } \\
\text { carbono acilico }\end{array}$ & 20 horas \\
\hline IV & $\begin{array}{c}\text { Estudo das reações } \\
\text { Quimicas }\end{array}$ & 25 horas & $\begin{array}{l}\text { Estrutura de sólidos } \\
\text { cristalinos e amorfos }\end{array}$ & 25 horas & $\begin{array}{l}\text { Reações de } \\
\text { substituição } \\
\text { eletrofilica em } \\
\text { aromáticos }\end{array}$ & 20 horas \\
\hline & & 80 horas & & 80 horas & & 80 horas \\
\hline
\end{tabular}

\begin{tabular}{|c|c|c|c|c|c|c|c|c|}
\hline \multirow[b]{2}{*}{ Unidade } & \multicolumn{8}{|c|}{ Ano } \\
\hline & Fisico Quimía & $\begin{array}{c}\mathbf{n}^{\circ} \text { de } \\
\text { horas aula }\end{array}$ & Quimica Analítica & $\begin{array}{c}\mathbf{n}^{\circ} \text { de } \\
\text { horas aula }\end{array}$ & $\begin{array}{c}\text { Análise } \\
\text { Instrumental }\end{array}$ & $\begin{array}{c}\mathbf{n}^{\circ} \text { de } \\
\text { horas aula }\end{array}$ & Corrosão & $\begin{array}{c}\mathrm{n}^{\circ} \text { de horas } \\
\text { aula }\end{array}$ \\
\hline I & $\begin{array}{c}\text { Solvçôes , Dispersões } \\
\text { e Propriedades } \\
\text { Coligativas }\end{array}$ & 25 horas & $\begin{array}{c}\text { Introduçāo, Tecnicas } \\
\text { e Métodos }\end{array}$ & 18 horas & $\begin{array}{c}\text { Introdução o aná lise } \\
\text { instrumental }\end{array}$ & 10 horas & Introd vção a corro são & 10 horas \\
\hline II & Equilibrio Quimico & 20 horas & $\begin{array}{l}\text { Titulometria de } \\
\text { neutralizaça ảo e } \\
\text { precipitaçäio }\end{array}$ & 20 horas & $\begin{array}{c}\text { Métodos } \\
\text { eletroan aliticos }\end{array}$ & 20 horas & $\begin{array}{c}\text { Princ pipios básicos da } \\
\text { corrosảo } \\
\text { eletroQuímica }\end{array}$ & 25 horas \\
\hline III & Cinética Quimica & 20 horas & $\begin{array}{l}\text { Titulometria de } \\
\text { complexsça âo e oxi- } \\
\text { redução }\end{array}$ & 23 horas & $\begin{array}{c}\text { Métodos } \\
\text { espectros cóp icos }\end{array}$ & 25 horas & $\begin{array}{c}\text { Princ ípios básicos da } \\
\text { corrosảo Quimica }\end{array}$ & 20 horas \\
\hline IV & $\begin{array}{c}\text { Estudo dos } \\
\text { Processos de Troca } \\
\text { de Calor nos } \\
\text { Equilibrios }\end{array}$ & 15 horas & Laboratónio, Cákulos & 19 horas & $\begin{array}{c}\text { Métodos } \\
\text { croms tográficos }\end{array}$ & 25 horas & $\begin{array}{l}\text { Res istênc ia à } \\
\text { corros ảo e proteçào } \\
\text { an ticorrosiva }\end{array}$ & 25 horas \\
\hline & & 80 horas & & 80 horas & & 80 horas & & 80 horas \\
\hline
\end{tabular}

RC: 78616

Disponível em: https://www.nucleodoconhecimento.com.br/education- 
DISCIPLINA CURRICULAR TÉCNICA - 3 ANO

\begin{tabular}{|c|c|c|c|c|c|c|}
\hline \multirow[b]{2}{*}{ Unidade } & \multicolumn{6}{|c|}{ Ano } \\
\hline & $\begin{array}{l}\text { Petróleo e } \\
\text { Polímeros }\end{array}$ & $\begin{array}{c}\mathbf{n}^{\circ} \mathrm{de} \\
\text { horas aula }\end{array}$ & $\begin{array}{l}\text { Processos } \\
\text { Qúnicos } \\
\text { Industriais }\end{array}$ & $\begin{array}{c}\mathbf{n}^{\circ} \text { de } \\
\text { horas aula }\end{array}$ & $\begin{array}{c}\text { Tecnologia de } \\
\text { Biocombustiveis }\end{array}$ & $\begin{array}{c}\mathbf{n}^{\circ} \text { de } \\
\text { horas aula }\end{array}$ \\
\hline $\mathbf{I}$ & $\begin{array}{c}\text { Process os } \\
\text { tecnológicos de } \\
\text { petróleo e polimeros e } \\
\text { Leg islação pertinente }\end{array}$ & 18 horas & $\begin{array}{l}\text { Tratamento de água, } \\
\text { Importancia do } \\
\text { Tratamento de } \\
\text { Efhentes, Parâmetros } \\
\text { de pohvição hidrica e } \\
\text { Classificação de } \\
\text { residuos }\end{array}$ & 25 horas & $\begin{array}{l}\text { Etapas Quimicas da } \\
\text { Sintese de Etanol e do } \\
\text { Biodiesel; Análise } \\
\text { Quimica de Qualidade } \\
\text { de Produção de Mini - } \\
\text { Usinas Pequeno, } \\
\text { Médio e Grande Porte }\end{array}$ & 25 horas \\
\hline II & $\begin{array}{l}\text { Controle de qualidade } \\
\text { e Fundamentos do } \\
\text { petróleo e dos } \\
\text { polimeros }\end{array}$ & 22 horas & $\begin{array}{c}\text { Tratamento e } \\
\text { disposição final de } \\
\text { efluentes de } \\
\text { residuo, Formas de } \\
\text { tratamentos, Tipos de } \\
\text { tratamento e descarte } \\
\text { e Tpos de } \\
\text { equipamentos } \\
\end{array}$ & 20 horas & $\begin{array}{c}\text { Produção e Análise } \\
\text { Quimica de } \\
\text { Biocombus tiveis em } \\
\text { Escala Laboratoriale } \\
\text { Industrial }\end{array}$ & 25 horas \\
\hline III & $\begin{array}{l}\text { Indústria do petróleo } \\
\text { e petroQuimica e } \\
\text { Logistica do petróleo }\end{array}$ & 22 horas & $\begin{array}{l}\text { Programas de } \\
\text { reutilização, } \\
\text { Resolução de } \\
\text { problemas de } \\
\text { produção e } \\
\text { qualidade de } \\
\text { a limentos, Otimização } \\
\text { na produção de } \\
\text { oleaginos as e açúcar }\end{array}$ & 20 horas & $\begin{array}{l}\text { Produção de Bio- } \\
\text { Etanol e Biodiesel }\end{array}$ & 10 horas \\
\hline IV & $\begin{array}{l}\text { Produção de } \\
\text { polimeros e } \\
\text { Reciclagem de } \\
\text { polimeros }\end{array}$ & 18 horas & $\begin{array}{c}\text { Otimização na } \\
\text { prodvção de álcool, } \\
\text { Oleaginos as da região } \\
\text { e Reações Quimicas } \\
\text { dos mais importantes } \\
\text { processos industriais }\end{array}$ & 15 horas & $\begin{array}{l}\text { Aspectos } \\
\text { operacionais de } \\
\text { usinas de etanol } \\
\text { biodiesele, } \\
\text { combustiveis de } \\
\text { Terceira geração }\end{array}$ & 20 horas \\
\hline & & 80 horas & & 80 horas & & 80 horas \\
\hline
\end{tabular}

La somme des questions de chimie ENEM entre 2014 et 2018, par degré de difficulté, par an apparaît dans la figure 1. Le nombre de questions de difficulté moyenne est passé de sept (7) en 2014 à douze (12) en 2015. Les deux années suivantes il y a eu une réduction, avec sept (7) questions en 2016 et cinq (5) en 2017. En 2018, il y a eu une légère augmentation, totalisant six (6) questions. En matière de grande complexité, il y a eu une réduction de dix (10) questions en 2014 à cinq (5) en 2015. Dès lors, il y a eu une augmentation les deux années suivantes,

RC: 78616

Disponível em: https://www.nucleodoconhecimento.com.br/education- 
avec dix (10) en 2016 et douze (12) ) en 2017 En 2018, il y a eu une légère baisse du nombre, onze (11) questions).

La figure 1 montre la somme des questions de chimie ENEM entre 2014 et 2018, par degré de difficulté, par année

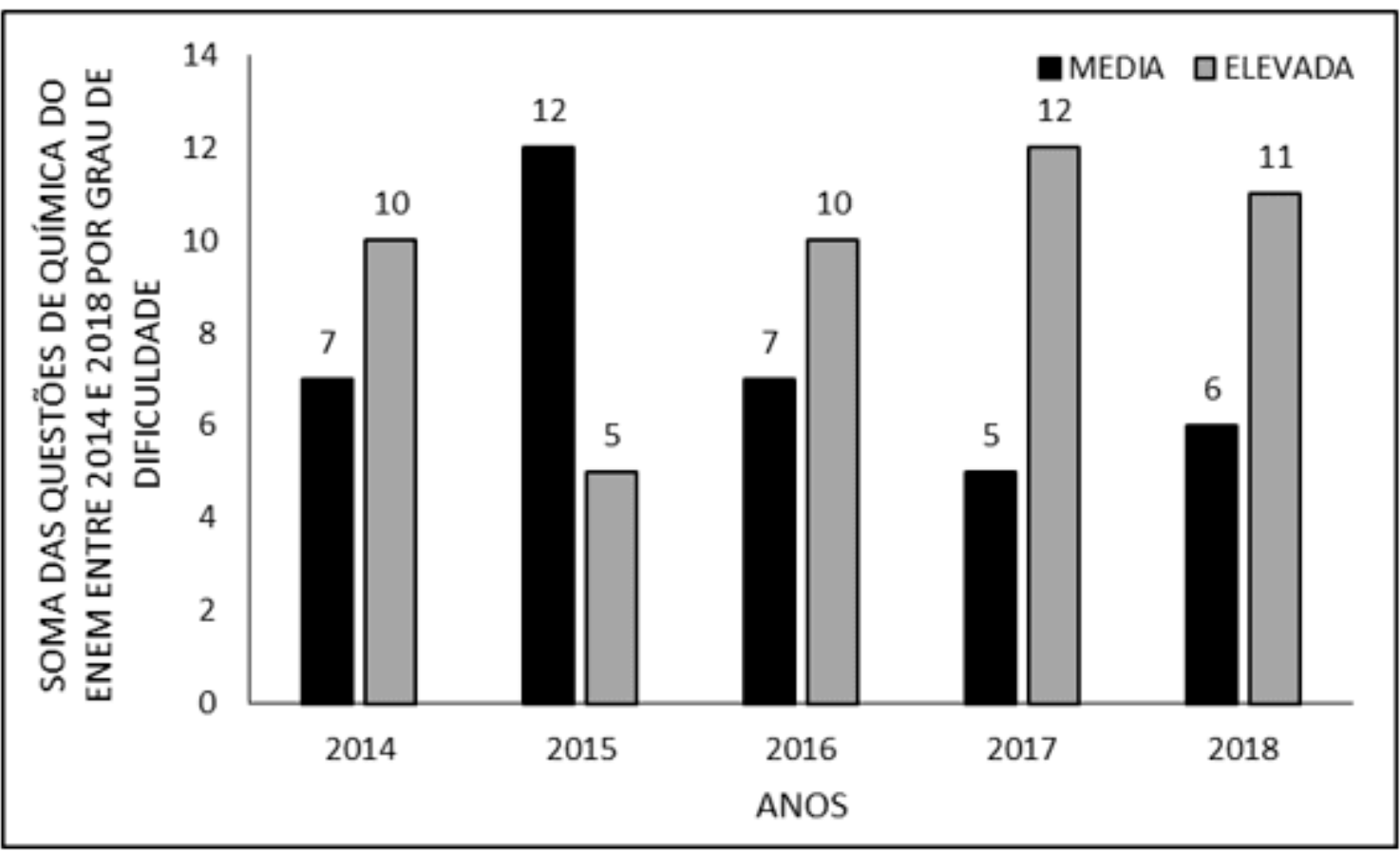

\section{DISCUSSION}

La différence présente dans les tests ENEM évalués, quant au pourcentage de chaque contenu (tableau 1) se produit probablement du fait que certains d'entre eux sont plus présents dans la vie quotidienne des élèves (comme les réactions organiques et les liens entre eux) que d'autres ( indicateurs, changements d'état, symbologie). ENEM valorise pour contextualiser ses questions (Hipólito et Silveira, 2011; Silveira et al., 2014).

Les contenus de l'ENEM se superposent car ils cherchent une intégration entre les thèmes internes de la matrice des cursus de chimie et entre ces thèmes et les autres

RC: 78616

Disponível em: https://www.nucleodoconhecimento.com.br/education- 
disciplines (interdisciplinarité et transversalité) (tableau 2). Ce mélange de contenus les aide à se rapprocher de la vie quotidienne des élèves. Cela aide également à changer le programme en quelque chose de moins plâtré et de plus transversal (Hipólito et Silveira, 2011).

Ce tableau montre également que la plupart des questions intradisplinaires concernent les enzymes, les réactions organiques, les solutions et la concentration des solutions. Cela est peut-être dû au fait que, physiologiquement, la plupart des êtres vivants fonctionnent en raison de la chimie de leur structure organique. Donc, encore une fois, des questions comme celle-ci sont plus proches du quotidien des étudiants (Leite et Velani, 2019).

Le contenu peut avoir des charges de travail différentes (tableau 3) en raison de différences de complexité et également de la capacité à contextualiser le contenu. La mise en contexte du contenu est importante pour leur compréhension et leur compréhension. Comme chaque enseignant a sa propre façon de les enseigner, il peut y avoir une différence entre le temps du programme programmé dans la planification et le temps réel pour leur enseigner (Pontes et al., 2008).

Le domaine des sciences naturelles a le plus grand poids pour le domaine de la santé et de la biologie dans la plupart des universités publiques du Brésil, comme I'USP, l'une des universités les plus prestigieuses du pays. Les cours dans le domaine de la santé sont très convoités, notamment en raison de leur rémunération. Les questions de chimie cherchent probablement à maintenir une difficulté plus élevée (Figure 1) afin que les candidats ayant plus de connaissances dans ce domaine de grande importance pour le cours entrent avec plus de connaissances dans ces domaines (Casoni, 2021; Ufpe, 2021).

RC: 78616

Disponível em: https://www.nucleodoconhecimento.com.br/education- 


\section{CONCLUSION}

Le contenu enseigné au cours des trois années du cours technique de chimie à I'Institut fédéral d'Amapá (IFAP) répond aux exigences de l'examen national du lycée (ENEM).

La charge de travail du cours technique de chimie du lycée à la IFAP est également suffisante pour le développement des disciplines de base et techniques, et fournit également une bonne base pour l'ENEM

L'analyse du contenu du cours a la chimie de la IFAP démontre que, comme il s'agit d'un cours technique, il fournit des connaissances approfondies, ce qui augmente la subvention pour l'achèvement de l'ENEM. Ce contenu est formé par la théorie et aussi par une grande expérience pratique (laboratoire). Les connaissances pratiques aident énormément à la fixation de l'apprentissage et fournissent des connaissances pour discuter du contenu.

Par conséquent, le contenu du programme de ce cours technique répond aux exigences de base de l'examen national.

\section{RÉFÉRENCES}

ALVES, N. B.; PALMA, L. C.; SILVA, T. N. Educação para a sustentabilidade: a construção de caminhos no Instituto Federal de Educação, Ciência e Tecnologia do Rio Grande do Sul (IFRS). Ram, Rev. Adm. Mackenzie, v. 14, n. 3, p. 83-118, 2013.

BRASIL. Matriz de Referência Enem. Brasilia DF, 2015. Disponível em: < http://download.inep.gov.br/download/enem/matriz_referencia.pdf $>$. Acesso em: 25 mar 2020.

RC: 78616

Disponível em: https://www.nucleodoconhecimento.com.br/education- 
. Curso Técnico de Nível Médio em Química na Forma Integrada Regime Integral: Plano de Curso. Macapá AP, 2016. Disponível em: < https://portal.ifap.edu.br/index.php/publicacoes/item/1100-resolucao-n-20-2018consup $>$.

. Expansão da Rede Federal. Rede Federal de Educação Profissional, Científica e Tecnológica. 2018. Disponível em: < http://redefederal.mec.gov.br/expansao-da-rede-federal >. Acesso em: 20 abr 2020.

Histórico. Macapá AP, 2019. Disponível em: < http://portal.ifap.edu.br/index.php/quem-somos/historico >. Acesso em: 24 abr. 2020. . Curso Técnico em Química - Integrado - Campus Macapá. Macapá AP, 2019a. Disponível em:

http://www.ifap.edu.br/index.php/component/content/article?id=398 >. Acesso em: 24 abr. 2020.

CASONI, L. A. SiSU Simulator. 2021. Disponível em: < https://sisusimulator.com.br/usp/faculdade-de-medicina-da-universidade-de-saopaulo_fm/medicina-bacharelado >. Acesso em: 24 fev 2021

CASTRO, G. N. V. et al. Análise de Eficiência Acadêmica dos cursos subsequentes, nas modalidades à distância e presencial, ofertados pelo Instituto Federal do Amapá (2018). Research, Society and Development, v. 9, n. 8, p. e208985262, 2020. https://rsdjournal.org/index.php/rsd/article/view/5262

HIPÓLITO, A. F.; SILVEIRA, H. E. D. As questões de Química do Exame Nacional do Ensino Médio (ENEM) em um enfoque transversal e interdisciplinar. 2011. Disponível em: < http://abrapecnet.org.br/atas_enpec/viiienpec/resumos/R0237-1.pdf >. Acesso em: 11 fev 2021.

$\mathrm{RC}: 78616$

Disponível em: https://www.nucleodoconhecimento.com.br/education- 
LEITE, K. D. C.; VELANI, V. Divertindo-se com a química: o ensino e a aprendizagem por meio do lúdico. Braz. J. of Develop., v. 5, n. 11, p. 25115-25133, 2019.

MARQUES, J. D. C. et al. Nível Médio Técnico e Cursos de Graduação: comparativo de vagas e ingressantes no Instituto Federal do Amapá, Brasil (20172018). Research, Society and Development, v. 9, n. 8, p. e228985375, 2020. https://rsdjournal.org/index.php/rsd/article/view/5375

MORETTO, M.; WITTKE, C. I. Capacidades de linguagem desenvolvidas em estudantes do ensino médio a partir de uma dinâmica de produção de textos focada no ENEM. Diálogo das Letras, v. 7, n. 2, p. 155 - 172, 2018.

PENHA, A. C. F. M. et al. Matrículas da Educação Especial na Educação Profissional Técnica de Nível Médio no Estado do Amapá (2015-2018). Research, Society and Development, v. 9, n. 7, p. e881974867, 2020. https://rsdjournal.org/index.php/rsd/article/view/4867

PONTES, A. N. et al. O Ensino de Química no Nível Médio: Um Olhar a Respeito da Motivação. XIV Encontro Nacional de Ensino de Química (XIV ENEQ) Curitiba PR: Universidade Federal do Paraná 2008.

SILVEIRA, F. L.; STILCK, J.; BARBOSA, M. Comunicações: Manifesto sobre a qualidade das questões de Física na Prova de Ciências da natureza no Exame Nacional de Ensino Médio. Caderno Brasileiro de Ensino de Física, v. 31, n. 2, p. 473-479, 2014.

UFPE. Relação dos Cursos com novos Pesos e Notas Mínimas do ENEM 2019 que serão aplicados no Processo Seletivo UFPE| SiSU 2020. 2021. Disponível em: < https://www.ufpe.br/documents/38970/2199517/Pesos+e+notas+m\%C3\%ADnimas+

$\mathrm{RC}: 78616$

Disponível em: https://www.nucleodoconhecimento.com.br/education- 
ENEM_UFPE+2020_+Mudan\%C3\%A7as+nos+cursos_03.06.19.pdf/85759192-f4cc4817-8541-026a5e06f7c4 >. Acesso em: 24 fev 2021.

Publié: Mars, 2021

Approuvé: Mars 2021

$\mathrm{RC}: 78616$

Disponível em: https://www.nucleodoconhecimento.com.br/educationfr/comparaison-des-programmes 